\title{
LETTERS TO THE EDITOR
}

\section{Rapid responses}

If you have a burning desire to respond to a paper published in $A D C$ or $F \mathcal{E} N$, why not make use of our "rapid response" option?

Log on to our website (www. archdischild.com), find the paper that interests you, click on "full text" and send your response by email by clicking on "submit a response".

Providing it isn't libellous or obscene, it will be posted within seven days. You can retrieve it by clicking on "read eLetters" on our homepage.

The editors will decide, as before, whether to also publish it in a future paper issue.

\section{Infantile colic and chiropractic spinal} manipulation

EdrTor,- We congratulate Olafsdottir et al on their article. ${ }^{1}$ The sum of the evidence on spinal manipulative therapy (SMT) in the treatment of infantile colic now is that there are 3 randomised controlled trials (RCTs) on the subject.

Two RCTs demonstrated a significant positive effect of SMT, ${ }^{23}$ and $1 \mathrm{RCT}$ was unable to demonstrate any treatment effect. ${ }^{1}$ The reasons for this discrepancy are not known, but Olafsdottir et al suggest that their finding of no effect of SMT may be due to the blinding of the infants' mothers. Another equally likely explanation could be that we are witnessing a dose response phenomenon.

In their trial, Olafsdottir et al used a treatment protocol of a maximum of 3 sessions of SMT, whereas the other 2 RCTs, which found a positive treatment effect, used a treatment protocol relying more on the treating chiropractor's clinical judgement. This more pragmatic approach resulted in $64 \%$ of the infants in one RCT receiving 4 or more sessions of SMT (with a maximum of 7), ${ }^{2}$ and the majority of infants in the other RCT receiving up to 6 sessions.

We believe that this dose response problem should be addressed in future trials of SMT for infantile colic.

\section{N GRUNNET-NILSSON University of Southern Denmark n.nilsson@imbmed.sdu.dk JESPER WIBERG Private practice, Copenhagen,} Denmark

1 Olafsdottir E, Forshei S, Fluge G, et al. Randomized controlled trial of infantile colic treated with chiropractic spinal manipulation. Arch Dis Child 2001;84:138-41.
2 Wiberg J, Nordsteen J, Nilsson N. The shortterm effect of spinal manipulation in the treatterm effect of spinal manipulation in the treatment of infantile colic: A randomized controlled trial with a blinded observer. 7 Manipulative Physiol Ther 1999;22:517-22.

3 Mercer C, Nook B. The efficacy of chiropractic spinal adjustments as a treatment protocol in the management of infantile colic. In: Haldeman S, Murphy B, eds. 5th Biennial Congress of the World Federation of Chiropractic. Auckland, New Zealand: 1999:170-1.

\section{Diluted treatment effects?}

EDITOR,-If my reading of this colic study is correct, it appears that both groups received standard counselling and recommendations for the care of a colicky child. My question to the authors is, if standard recommendations are effective in the reduction of colic, does this not raise the possibility that any treatment effect in the SMT group could have been diluted by the introduction of a second active treatment (standard recommendations) in the control group? Put another way, was the placebo intervention an inert intervention or was it a second active intervention?

G W KUKURIN D C DACAN Pittsburgh, PA, USA doc@alt-compmed.com

\section{Commentaries_read with caution!}

EDITOR,- The commentary by Lenney correctly points out that clinicians are often slow to apply good research evidence to clinical practice. ${ }^{1}$ However, the choice of once daily intravenous gentamicin to illustrate this point is unfortunate. Extended interval aminoglycoside dosing is widely used in paediatric and neonatal practice for the treatment of serious gram negative infections, the treatment of newborn infants with sepsis, and the treatment of chronic Pseudomonas aeruginosa infection in patients with cystic fibrosis. However, the implementation of extended interval dosing has not been based on the results of appropriately designed trials in children and neonates.

The largest meta-analysis of single versus multiple daily dosing of aminoglycosides for the treatment of gram negative sepsis included only 2 paediatric studies. ${ }^{2}$ The use of once daily aminoglycosides in children and the newborn is still currently unlicensed. Finally, a recent systematic review of once daily versus multiple daily dosing of aminoglycosides in CF concluded that there was insufficient evidence to recommend a change in practice. ${ }^{3}$ This was because most clinical trials were of insufficient quality or were performed in adults and so the results should not be extrapolated to children.

We argue that the presence of evidence from "a number of studies from numerous countries" should not be the basis on which implementations in practice should be founded. Instead, quality of evidence should be of paramount importance, even if there is little of it.

K TAN Academic Division of Child Health, University of Nottingham, Nottingham, UK Kelvin.Tan@nottingham.ac.uk A SMYTH Nottingham City Hospital NHS Trust, Nottingham, UK

1 Lenney W. Successful implementation of spacer treatment guidelines for acute asthma. Arch Dis Child 2001;84:145-6.
2 Barza M, Ioannidis JPA, Cappelleri JC, et al. Single or multiple daily doses of aminoglycosides: gle or multiple daily doses of aminoglyco

3 Tan K, Bunn H. Once daily versus multiple daily dosing with intravenous aminoglycosides for cystic fibrosis (Cochrane Review). Cochrane Database Syst Rev 000;4:CD002009 2000; CD0020099.

Alcopops are not responsible for acute paediatric attendances with alcohol intoxication

EDITOR, - We were interested to read Dr Robson's leading article regarding alcohol misuse and the reference to acute alcohol admissions to Alder Hey in Liverpool, UK. ${ }^{1}{ }^{2}$ We too are concerned by the increasing number of these problems that we see in hospital paediatric practice.

We carried out a retrospective case note review of all the children seen in the Paediatric Emergency department in Sunderland between November 1999 and October 2000. One hundred children ( 57 female) accounted for 106 attendances with acute alcohol intoxication ( 2 children attended twice and 2 three times). The notes of 97 attendances were available for review. Most children were aged 13 to $15(77 \%)$, range $10-16$ years. As might be expected, the majority presented during the weekend (66\%) and in the evening or at night ( $84 \%$ between 19:00 and 01:00). Half had been drinking with friends in a public place, although precise details were not recorded in many cases. Sixty one children $(63 \%)$ were brought in by emergency ambulance and $48(49 \%)$ were admitted. Thirty (31\%) were documented to have been drinking vodka, 21 cider $(22 \%), 12(12 \%)$ beer or lager, $11(11 \%)$ other spirits, $8(8 \%)$ wine, and $8(8 \%)$ a combination of these. The type of alcohol was not recorded in $7(7 \%)$ cases. In no cases were alcopops thought to be the beverage responsible for the acute attendance, and the beverages consumed are comparable with Alder Hey figures from $1996 .^{2}$

Alcopops and designer drinks appeal to young people, particularly $14-16$ year olds, and there has been criticism that marketing may be aimed at this age group. ${ }^{34}$ Consumption of alcopops has been associated with drinking in less controlled environments, heavier drinking, and greater self reported drunkenness. ${ }^{3}$ However, our data do not suggest that they are a problem in relation to acute intoxication presenting to Accident and Emergency. We support the statement that children will mimic adults in their use and misuse of alcohol, and consider that it is society's changing attitude to alcohol and not the type of alcohol available that is of concern.

D CROSSLAND K POTIER DE LA MORANDIERE Department of Paediatrics, Sunderland Royal Hospital, Kayll Road, Sunderland SR4 7TP UK davidxland@hotmail.com

Robson WJ. Alcohol misuse. Arch Dis Child 2001;84:95-7.

2 Robson WJ. Alcohol and adolescents. Fournal of Substance Misuse 1998;3:3-4.

3 Hughes K, MacKintosh AM, Hastings G, et al. Young people, alcohol, and designer drinks: quantitative and qualitative study. BMf 1997; 314:414-18

4 McKeganey N, Forsyth A, Barnard M, et al. Designer drinks and drunkenness amongst a sample of Scottish schoolchildren. BMF 1996; 313:401. 


\section{Other implications of disposable nappies}

EDITOR,-Partsch, Aukamp, and Sippell propose that increased testicular temperature in early childhood might affect later spermatogenesis. They suggest that disposable nappies could contribute to this and demonstrate a significant difference between the scrotal skin temperature recorded in infants using disposable nappies and washable cotton nappies. They mention in their introductory paragraph that other environmental factors may be important in the deterioration seen in male reproductive health over recent years, but do not relate any of these factors to disposable nappies.

There are many concerns about the use of disposable nappies in addition to increasing scrotal temperature that may impact on future fertility and general health. The disposable nappy consists of a plastic outer layer, a layer of superabsorbent chemicals and inner liner. Nappies are not subject to government controls or independent testing and disposable nappy manufacturers do not need to disclose the contents. ${ }^{23}$

Recently, concern has been raised about the presence of Tributyl Tin (TBT) in disposable nappies. Greenpeace and Women's Environmental Network have commissioned research which showed that there were significant levels of TBT in many brands of disposable nappy, including those on sale in the UK. ${ }^{45}$ Babies may be in contact with up to 3.6 times the WHO's estimated tolerable daily intake. TBT is an environmental pollutant which is used in anti-fouling ship paint. It is known to disrupt the endocrine and immune function of marine shellfish and there are international plans to phase out its use.

The superabsorbent chemicals used include sodium polyacrylate crystals which form a gel in contact with urine. This gel can be seen on the skin in contact with it and there are particular concerns about this entering the body through broken skin in the nappy area. Sodium polyacrylate, along with other chemical constituents that increase absorbency, has been removed from tampons as it was associated with the development of Toxic Shock Syndrome. ${ }^{6}$ The inner liner has previously been shown to contain nonylphenyl ethoxylate, which acts as an oestrogen mimic, and dioxins. ${ }^{3}$

In addition, the use of disposable nappies has important environmental consequences which may impact on child health. Manufacture of disposable nappies uses 3.5 times more energy, 8 times as many non-renewable resources, and 90 times as many renewable resources when compared with washable nappies. The description of such nappies as "disposable" is misleading. In this country, nappies make up approximately $4 \%$ of household waste $(800000$ tonnes per year) and every disposable nappy and its contents ever used is still present in a landfill site. ${ }^{2}$
There are environmentally friendly and safe alternatives to the disposable nappy. Modern washable nappies are very different from the traditional idea of buckets of "terries". There are now shaped cotton nappies with velcro fastenings, alternatives to nappy pins, breathable covers, and disposable paper inner liners. Concern that the incidence of nappy rash is higher with washable nappies is unfounded-it has been shown that it is the length of contact of urine with the skin that is most important in the development of nappy $\operatorname{rash}^{7}$ and it may be that an infant in a disposable has more chance of developing nappy rash as they are often changed less frequently than an infant in washable nappies. In addition, there are cost savings both to individuals and organisations using washable nappies, and there have been several successful hospital projects using washable nappies on postnatal wards. ${ }^{2}{ }^{3}$

As paediatricians committed to the health of children, we should be aware of the issues raised by the use of disposable nappies, the alternatives that exist, and sources of information and support for parents who are concerned about ensuring a safe and sustainable future for their children.

C HEAL C COOPER

Consultant Paediatricians, Royal Albert Edward Infirmary, Wigan Lane, Wigan WW1 2NN, UK

1 Partsch C-J, Aukamp, Sippell WG. Scrotal temperature is increased in disposable plastic lined nappies. Arch Dis Child 2000;83:364-8.

2 Link A. Preventing Nappy Waste. London: Women's Environmental Network, 1996.

3 Sustainable Wales. Dumping the Diaper! Reusable Nappy Report.

4 Greenpeace. Greenpeace calls on parents to return contaminated nappies to producers: new tests contaminated nappies to producers: new tests
show that TBT-free nappies are a rarity. Press Release 19th May 2000 www.greenpeace.org.

5 Women's Environmental Network. Press Release 300700 . 30th July 2000 www.realnappy.com. 6 Reingold AL. Toxic shock syndrome: an update. Am ₹ Obstet Gynecol 1991;165:1236-9.

7 Philipp R, Hughes A, Golding J. Getting to the bottom of nappy rash. ALSPAC Survey Team. Avon Longitudinal Study of Pregnancy and Childhood. Br 7 Gen Pract 1997;47:493-7.

\section{Dexamethasone, survival, and} neurological impairment

EditoR,-Professor Pharoah questions whether the increased rate of cerebral palsy among newborn infants who were randomly allocated early postnatal dexamethasone therapy in the trial by Shinwell et al might be because dexamethasone increased survival of infants who were impaired before birth, and not because dexamethasone caused cerebral impairment.

However, two recent systematic reviews of randomised trials of postnatal dexamethasone therapy in infants at risk of chronic lung disease do not support this hypothesis. Halliday and Ehrenkrantz found no difference in survival in trials of dexamethasone given within 96 hours of birth. ${ }^{2}$ Doyle and Davis found no difference in survival, overall or in any subgroups, in trials of dexamethasone therapy at any time after birth. ${ }^{3}$ Both reviews concluded that postnatal dexamethasone may cause neurological dysfunction and called for further trials with appropriate follow up.

Professor Doyle is currently co-ordinating such a trial in infants under $1000 \mathrm{~g}$ or less than 29 weeks who are ventilated after 7 days from birth (the DART study, Dexamethasone in tiny infants-a Randomised Trial). Those interested in participating in this important study are very welcome to contact him at1.doyle@obgyn-rwh.unimelb.edu.au.

W TARNOW-MORDI

Westmead Hospital and The Children's Hospital at Westmead,

University of Sydney, Australia lwd@unimelb.edu.au

1 Shinwell ES, Karplus M, Reich D, et al. Early postnatal dexamethasone treatment and incidence of cerebral palsy. Arch Dis Child Fetal Neonatal Ed 2000;83:F177-81.

2 Halliday HL, Ehrenkranz RA. Early postnatal ( $<96$ hours) corticosteroids for preventing chronic lung disease in preterm infants. (Cochrane Review). In: Cochrane Library, Issue 1, 2001. Oxford: Update Software.

3 Doyle L, Davis P. Postnatal corticosteroids in preterm infants: systematic review of effects on preterm infants: systematic review of effects on Health 2000;36:101-7.

\section{NOTICE}

\section{Notice of duplicate publication}

Rushforth H, Bliss A, Burge D, Glasper E. A pilot randomised controlled trial of medical versus nurse clerking for minor surgery. Archives of Disease in Childhood 2000;83: 223-6.

Rushforth H, Bliss A, Burge D, Glasper E. Nurse led pre-operative assessment: a study of appropriateness. Paediatric Nursing 2000;12(5):15-20.

The same data, resulting from a single pilot study were reported in the two above papers. The authors have apologised, explaining that they had not intended to flaunt accepted academic standards, rather that they wished to bring their findings to the attention of two separate readerships-namely paediatricians and nurses. However, we would not wish compilers of systematic reviews to include these data twice and therefore we give notice of duplicate publication and withdraw the article published in Archives of Disease in Childhood. 